\title{
Horizon-T extensive air showers detector system operations and performance
}

\author{
D. Beznosko', A. Batyrkhanov, T. Beremkulov, A. Duspayev, A. lakovlev, Z. \\ Makhataeva, K, Yelshibekov, M. Yessenov \\ Nazarbayev University \\ 53 Kabanbai Batyr ave, Astana, KZ \\ E-mail: dmitriy.beznosko@nu.edu.kz
}

\section{R. U. Beisembaev, V. V. Zhukov}

P. N. Lebedev Physical Institute of the Russian Academy of Sciences

53 Leninskiy Prospekt, Moscow, Russia

\begin{abstract}
"Horizon-T" is an innovative detector system located at Tien Shan high-altitude Science Station (TSHASS) at approximately 3340 meters above the sea level. It consists of eight detection points separated by the distance up to one kilometer that can measure time characteristics of the Extensive Air Showers (EAS) and record signal shapes with time resolution of 10 ns. It was constructed to register EAS in the energy range above $10^{16} \mathrm{eV}$ coming from a wide range of zenith angles $\left(0^{\circ}-85^{\circ}\right)$. The system includes both the plastic scintillator particle detectors as well as the Vavilov - Cerenkov radiation detectors subsystem to observe the Cerenkov light from the EAS in the atmosphere directly. The time resolution and signal shape analysis capabilities of the detection points are used to study EAS development in the atmosphere.

The development of the EAS is a process that can be studied both spatially and temporally. For the spatial part, a distributed network of detection points is required. For the time part, a signal shape must be recorded and analysed at each point with time resolution on the order of $\sim 10 \mathrm{~ns}$. In this paper, the current system description and performance level are described. Additionally, the latest data examples showing the unusual EAS examples above $10^{17} \mathrm{eV}$ are included.
\end{abstract}

38th International Conference on High Energy Physics

3-10 August 2016

Chicago, USA

${ }^{1}$ Speaker 


\section{Introduction}

The Horizon-T (HT) detector system [1] realizes ideas that were first formulated in [2] and [3]. The first results of the Horizon-T have been published in [4] and [5] with another publication being prepared currently.

As EAS develops while passing through the atmosphere, ultra-relativistic electrons, muons and Vavilov - Cerenkov radiation photons each form a shower disk. Calculations, carried using CORSIKA [6] EAS simulation software package, indicate that for vertically incoming EAS with primary particle energy $\sim 10^{17} \mathrm{eV}$ at the distance $100 \mathrm{~m}$ from the core the charged particles pass the observation level in $\sim 15-20$ ns and in a few ns near the core. This sets minimal time resolution scale for HT and it is currently at better then $\sim 10 \mathrm{~ns}$.

With the increase of zenith angle the apparent thickness of the shower disks increases. The ability to conduct measurements at large zenith angles near to the horizon and the high time resolution give the name of the experiment: Horizon- $\mathrm{T}$ (where $\mathrm{T}$ stands for time). Developing on the HT ideas, there is also a project for a more advanced detector HT-KZ [7] (HTKazakhstan) to be constructed in the near future.

\subsection{Current Horizon-T}

The TSHASS was established in 1958 as a station devoted to studying of the cosmic rays in a wide energy range from the galactic and extra-galactic sources. Since then, the station personnel has accumulated a vast amount of knowledge and experience and has participated in a large number of different ground-breaking experiments over the course of the years. The study of time characteristics of the muon component was also carried our for many years.

The current HT system consists of three Vavilov - Cerenkov radiation detector (VCD) (Figure 1, left) and eight working charged particles detection points (Figure 1, right) that are separated up to $500 \mathrm{~m}$ distance from the center point.
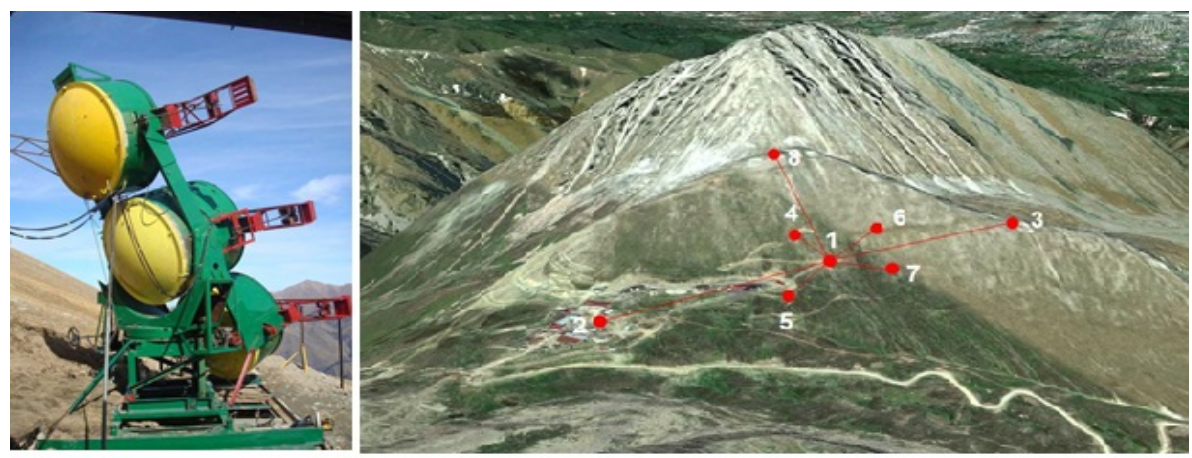

Figure 1: Horizon-T detector: Cerenkov light detectors (left) and charged particles detection points location (right)

The methodology of the current HT detector system is to look at the EAS near the horizon (up to $85^{\circ}$ from zenith) and to obtain the coordinates of the incoming EAS primary particle using the timing resolution from the disk reaching the detector. In addition, the VCD provides the detection of the Vavilov - Cerenkov light from the charged EAS components (EM and muons). 


\section{HT Components}

Each detection point except point 8 have three scintillator detectors (SD), oriented perpendicular to each other in the $\mathrm{x}, \mathrm{y}$ and $\mathrm{z}$ planes. Point 8 that has only the z-plane one. The $\mathrm{z}-$ plane is parallel to the sky. This arrangement is needed for the angular isotropy in the registration of charged particles. SD use polystyrene-based square-shaped cast scintillator [8] with $1 \mathrm{~m}^{2}$ area and $5 \mathrm{~cm}$ width. SD have either PMT-49 (FEU49B) [9] or Hamamatsu [10] R7723 PMTs. Liquid scintillator is considered as a possible upgrade [11].

The VCD is located next to detection point 1. It consists of three parabolic mirrors of 150 $\mathrm{cm}$ diameter and focal length of $65 \mathrm{~cm}$. They are mounted on the rotating support allowing detection in zenith angle range of $0^{\circ}-80^{\circ}$ and in azimuthal angle range of $0^{\circ}-360^{\circ}$. РМТ-495 (FEU49B) that is $15 \mathrm{~cm}$ in diameter is located in the focal point of each mirror. The field of view of each mirror + PMT is $\sim 13^{\circ}$.

From the geographical regions studied for the astroclimate, eastern Tien-Shan is well suited for Vavilov - Cerenkov radiation measurements [12] since for the most of the year there is a Rayleigh - type atmosphere when there is no aerosol present.

HT data aquisition system uses three CAEN[13] DT5730 flash ADCs with syncronization and self-trigger using the SD signals.

\section{Unusual EAS signals at HT}

CORSIKA [6] simulation software is based on our current understanding of HEP, thus simulating a 'standard' shower. At observation level, such EAS has a single disk with particle density changes approx. $1 / r^{2}$ from the core and passage time is growing $\sim r^{2}$ as well. An image of a typical event signal is shown in Figure 2.

FADCData from Jan26230336.root

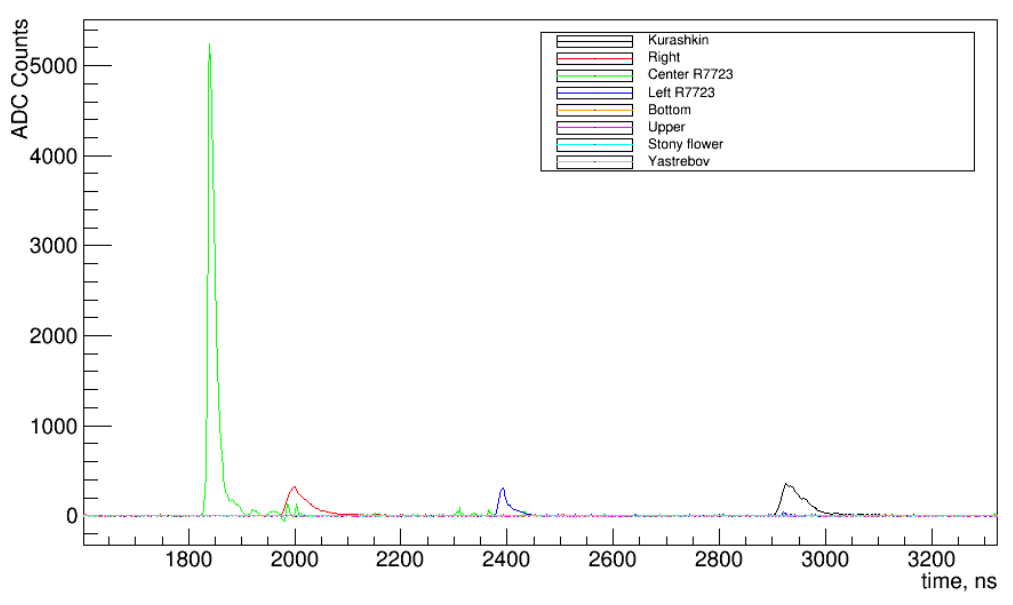

Figure 2: Image of a typical event at HT detector system.

Below are the examples of 'unusual' EAS signals. Note that all HT hardware has been calibrated [14] to ensure signal purity and proper functionality, and to exclude signal artefacts.

When first observed by J. Jelly and W. Whitehouse [15], they were attributed to delayed particle effect at the time. At HT, these signals are called 'multimodal'. The HT superior time 
resolution and full signal shape analysis indicates the attribution of the multimodal EAS to possible new phenomena. This is a work in progress and the results will be reported separately.

The examples of the 'unusual' EAS signals are shown in Figure 3 and Figure 4. Diffrent detection points have signals that may come from multiple disks arriving at lartge time differences. If this were due to delayed particle, a mass of a particle created needs to be unreasonably large thus this explanation of the effect observed is highly unlikely. All these events are above $\sim 10^{17} \mathrm{eV}$ due to the area covered by the disk (e. g. number of the points hit)

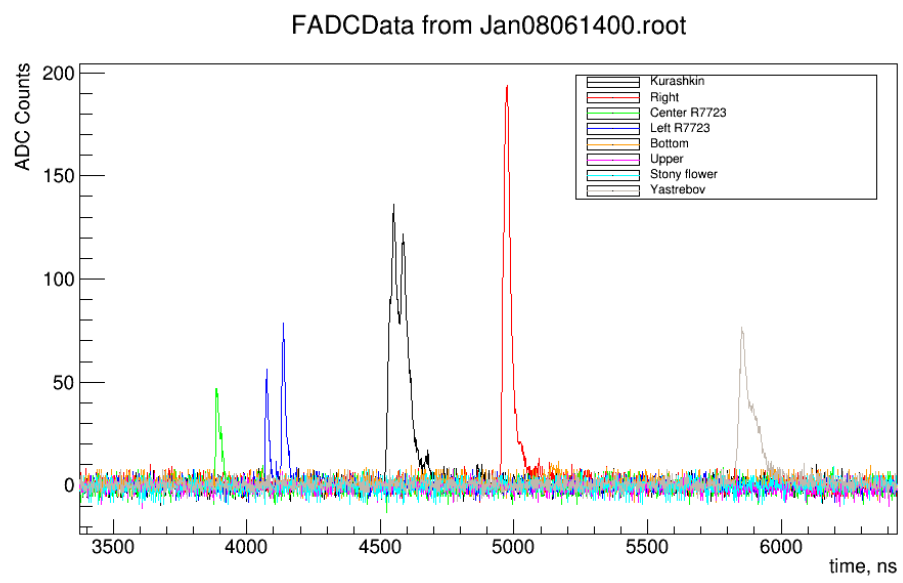

Figure 3: Example of the 'unusual' EAS signal.

FADCData from Jan09041819.root

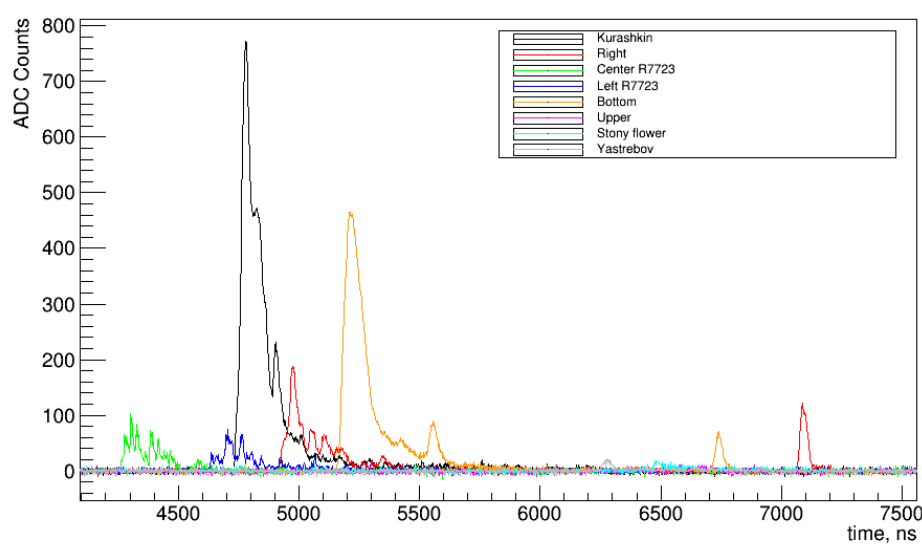

Figure 4: Example of the 'unusual' EAS signal.

\section{Conclusion}

Horizon-T is a new EAS detector taking advantage of both the spatial and temporary distribudion of the charged particles in a disk. Its capabilities allow to see 'unusual' EAS signals appearing at the higher end of the energy spectrum available to HT. 


\section{References}

[1] RU Beisembaev et al., The 'Horizon-T' Experiment: Extensive Air Showers Detection, arXiv:1605.05179 [physics.ins-det], May 172016.

[2] R. U. Beisembaev, Detector system project for studies of EAS at energies above $10^{17} \mathrm{eV}$. AS News, KZ Rep, phys-math series, \#6 C. pp 74-76 (1991).

[3] [R.U. Beisembaev et al., Muons of extra high energy horizontal EAS in geomagnetic field and nucleonic astronomy. Proc. 24 ICRC. Roma. 1. pp 646-649 (1995).

[4] R.U. Beisembaev et al., Observations of the Cerenkov light and the charged particle of the EAS at large zenith angles. Bul. of RAS, 2011, volume 75 (3), p 385 (2011).

[5] R. U. Beisembaev et al., The first results obtained with the HORIZON-T detector system, Journal of Physics. 409. 012127. (2013).

[6] D. Heck et al., CORSIKA: A Monte Carlo Code to Simulate Extensive Air Showers, Forschungszentrum Karlsruhe Report FZKA (6019)

[7] A. Duspayev at al., The distributed particle detectors and data acquisition modules for Extensive Air Shower measurements at "Horizon-T KZ" experiment, in proceedings to PhotoDet2015 conference PoS(PhotoDet2015)056.

[8] A Baitenov et al., Technical manual: a survey of scintillating medium for high-energy particle detection, arXiv:1601.00086, 2016/1/1

[9] MELZ-FEU, 4922-y pr-d, 4c5, Zelenograd, g. Moskva, Russia, 124482 (http://www.melz-feu.ru).

[10] HAMAMATSU PHOTONICS K.K., Electron Tube Division, 314-5, Shimokanzo, Iwata City, Shizuoka Pref., 438-0193, Japan, http://www.hamamatsu.com

[11] L. J. Bignell et al., Characterization and Modeling of a Water-based Liquid Scintillator, Journal of Instrumentation, Vol. 10, p 12009, IOP Publishing (12/2015)

[12] T. B. Omarov et al., Astroclimate of high altitude plateau Assy-Turgen, Almaty: Science 1982, pg 60.

[13] CAEN S.p.A. Via della Vetraia, 11, 55049 Viareggio Lucca, Italy. http://caen.it.

[14] T Beremkulov et al., Horizon-T Experiment Calibrations-Cables, arXiv:1608.04312, 2016/8/15

[15] J.V. Jelly and W.J. Whitehouse. Proc. Phys. Soc. (London) A66 454 (1953). 